\title{
Atherothrombosis in South Asians: Implications of Atherosclerotic and Inflammatory Markers
}

\author{
Sunita Dodani* \\ Department of Internal Medicine, University of Kansas Medical Center, Kansas City, USA
}

\begin{abstract}
South Asian immigrants (SAIs) have a higher prevalence of cardiovascular (CV) morbidity and mortality compared with other populations. The major challenge associated with primary prevention of cardiovascular to coronary artery diseases (CAD) in SAIs involves early and accurate detection of CAD in asymptomatic individuals at high cardiovascular risk. Inflammatory processes are now recognized to play a central role in the pathogenesis of atherosclerosis and are found to be associated with future $\mathrm{CV}$ risk in a variety of clinical settings. Imaging measures, such as common carotid artery intima-media thickness (CCA-IMT), are being applied as surrogate markers for end-points, such as myocardial infarction (MI) and death in clinical trials. Considering high CAD risk in SAIs and knowing that conventional risk factors may not fully explain the excess CAD risk in this group, studies on the role of CCA-IMT in CAD prediction have been discussed. Also, C-reactive protein (CRP) validity in risk prediction, the role of dysfunctional high density lipoprotein (HDL) as a CAD risk marker in SAIs have been presented.
\end{abstract}

Keywords: Coronary artery disease, Cardiovascular disease, Dysfunctional HDL, South Asians, C-reactive protein.

\section{INTRODUCTION}

Cardiovascular diseases (CVDs) account for more than 15 million deaths each year in the world [1]. Many who die are under the age of 65 and given today's increased life span, these deaths are premature. South Asians are individuals whose ethnic roots originate from the Indian subcontinent, a large geographic area that includes India, Pakistan, Sri Lanka, Nepal, and Bangladesh. Collectively, South Asians represent one fifth of the global population [2-4]. In North America, more than 3.6 million South Asians live in the US, and although this group represents the second fastest growing Asian immigrant population, little is known regarding their increased risk for CAD. It is important to recognize that the term "South Asian" refers to a heterogeneous population, with important differences in diet, culture, and lifestyle among different South Asian populations and religions. Multiple studies of migrant South Asian populations have, however, confirmed a 3- to 5-fold increase in the risk for myocardial infarction (MI) and cardiovascular death as compared with other populations [4-6]. Studies in the United Kingdom (UK) have found that South Asian Immigrants' (SAIs) risk of CAD death is as much as $40 \%$ above whites' $[7,8]$. In the US, South Asian Indian men have been found to have a rate of heart attacks that is nearly double than the general US population i.e. $7 \%$ vs. 3\% [9]. Studies comparing CAD risk factors amongst Indians living in India to immigrants in Britain [10] highlight that CAD risk factors are markedly higher amongst Indian Punjabi [11] and Gujarati [12] migrants than their counterparts in India. Traditional CV

*Address correspondence to this author at the Department of Internal Medicine, University of Kansas Medical Center, 3901 Rainbow Blvd. Kansas City, Kansas 66160; Tel: 913-588-6000; Fax: 913-945-7008;

E-mail: sdodani@kumc.edu risk factors like hypertension, smoking or high cholesterol do not fully account for the increased CAD risk in SAs. Insulin resistance is thought to be a contributory factor, but the exact patho-physiological mechanism for these increased incidences in SAs awaits further insight. A study comparing SA men to European Caucasian men showed elevated systemic arterial stiffening in SA men that could be partly responsible for the high CVD burden in this population. Increased insulin resistance may lead to arterial stiffening. Vessel wall composition in SAs may be another factor playing a role [13]. Knowing the high prevalence of CAD and its risk factors in South Asian immigrants, a major challenge associated with primary prevention of CAD involves early detection of CAD in those individuals who are at risk but are asymptomatic. Inflammatory mechanisms play a prominent role in mediating all stages of atherosclerosis, and measurement of inflammatory biomarkers provides a method for detecting individuals at future vascular risk. Advances in cardiovascular imaging, especially with reference to common carotid artery intima media thickness (CCA-IMT), have the potential to improve the early detection of atherosclerotic vascular disease and quantify its progression. Clinicians and their patients are challenged with how best to integrate these emerging modalities into clinical practice and understand their implications as surrogate markers for clinical end points in trials.

We therefore, present here a review of literature and updates on studies examining the role CCA-IMT in CAD prediction. In addition, we have briefly touched upon implications of C-reactive protein (CRP) and its validity in CAD risk prediction especially in SAIs. At the end of the review, we have provided insights on the dysfunctional HDL, and its potential role as CAD risk marker. 


\section{COMMON CAROTID ARTERY INTIMA-MEDIA THICKNESS (CCA-IMT)}

Assessment of CCA-IMT is well-recognized as a surrogate marker of atherosclerosis [14-20]. Moreover, CCA-IMT is a strong and independent predictor of death, stroke and myocardial infarction (MI) in hypertensive patients with CAD referred for coronary angiography. CCA-IMT is one of the methods for the detection of early stages of atherosclerotic disease and has been chosen as a surrogate marker over other non-invasive methods because it is a high-resolution, noninvasive technique that is readily available, allows to visualize the vessel wall structure with high resolution [21]. Pignoli and others established that the distance measured between two lines separated by a hypoechogenic space visible in an ultrasound image of the carotid distal wall correlated with the thickness of the intima-media (IMT) layers of the artery measured in vitro and in vivo [22]. Later studies extended this measurement to the thickness of the focal carotid plaque, and this "composite thickness" was used as a marker of atherosclerotic burden. This method has been widely used since its introduction, and validated in many cross-sectional and longitudinal epidemiologic and clinical studies. In addition, carotid atherosclerosis (IMT $>1.0 \mathrm{~mm}$ ) was associated with severe coronary disease (odds ratio, 2.2; $95 \%$ confidence interval, 1.2 to 4.0 ) in subjects undergoing elective coronary angiography [23]. The American Society of Echocardiography's (ASE) 2008 consensus statement on CCA-IMT defines it as the combined thickness of the intimal and medial layers of the far arterial wall of the carotid artery [24]. Carotid plaque is defined as focal arterial wall thickening $50 \%$ greater than the surrounding wall or a focal region of CCA-IMT $>1.5 \mathrm{~mm}$ [25]. Although standard carotid duplex ultrasonography is primarily used to identify occlusive carotid plaque (advanced atherosclerosis), CCA-IMT assessment measures arterial wall thickening (pre-atherosclerosis) and non-occlusive plaque formation (subclinical atherosclerosis).

According to data published after the completion of major epidemiologic studies as the Atherosclerosis Risk in Communities (ARIC) Study [25, 26], the European British Heart Study [27] and the Rotterdam Study [28], the increase in IMT not only correlates with most of the known atherosclerotic risk factors such as systolic hypertension, total and Low Density Lipoprotein (LDL) cholesterol levels, cigarette smoking, high-sensitivity C-reactive protein (hsCRP) levels, diabetes, and others, but also increases the risk of cardiovascular events. The results of the ARIC Study show that with an increase of $0.19 \mathrm{~mm}$ of the IMT, the risk of coronary disease increases by $69 \%$ in women and $36 \%$ in men $[25,26]$. The risk of stroke is 8.5 times higher for women and 3.6 times higher for men with IMT $>1 \mathrm{~mm}$, compared with those with IMT $<0.6 \mathrm{~mm}$.

In classic screening procedures, there is a strong tendency to concentrate on modifiable risk factors, especially when the data collection is relatively simple and easily available (e.g., blood samples or blood-pressure measurements). However, when patients are treated actively, those classic risk factors can lose their predictive value. Although levels of known CV risk factors vary in South Asians, they do not fully explain the differences in CAD rates [29]. The traditional approach to CAD risk assessment is based on identify- ing and to a certain extent quantifying established CV risk factors. Several algorithms based on this approach are used $[30,31]$. Among these, the Framingham Risk Assessment Model is the most widely accepted. Although used extensively and generally accepted, this model (as well as other algorithms based on similar approaches) has limitations. It is derived from a white Caucasian population in the US and may be less applicable to other ethnic groups. Family history, abdominal adiposity, inflammation, CCA-IMT and other factors shown to predict CV risk [31-33] are not incorporated in the Framingham risk score (FRS). Diabetes and smoking are identified only as present or absent, although current evidence supports a continuous relationship between glycemia and tobacco exposure to CAD risk [34, 35]. Age is the overriding FRS determinant, ignoring the greater interindividual variation in atherosclerotic burden at older ages and often providing false reassurance at younger ages. Importantly, the FRS only predicts short-term (10-year) risk, although from a clinical perspective the life-long risk of developing CAD events is equally relevant [36]. Early detection of sub-clinical CAD in high risk South Asian at a young age could help prevent $\mathrm{CV}$ events and substantially reduce the level of death and disability attributable to CAD. Imaging of arteries to identify and quantify the presence of subclinical atherosclerosis has been suggested to further refine $\mathrm{CV}$ risk assessment. Limited published data is available looking into the associations of CV factors with CCA- IMT in South Asians, especially in SAI groups. Anand et al. performed a cross-sectional population study in 1015 Canadian adults of Caucasian European, South Asian, Chinese and Aboriginal ancestry [35]. This study showed that $22 \%$ of SAIs who were categorized as low risk based on FRS had CCA IMT values of $\geq 75$ th percentile for age, sex and ethnicity. Furthermore, CCA-IMT has been shown to be independently associated with CAD in South Asians [36] and is a reproducible clinical tool to evaluate atherosclerosis, predict coronary artery disease and show the effectiveness of medical therapies $[30,37,38]$. We have shown in several of our studies on SAIs in the US that even without CAD or diabetes, positive CCA-IMT ( $\geq 0.8 \mathrm{~mm}$ ) was seen in $40 \%$ of homogeneous multi-ethnic SAI groups [39]. Moreover, positive CCA-IMT was also found to be associated with dysfunctional HDL (Dys-HDL) after adjusting for age, family history of cardiovascular disease, and hypertension $(\mathrm{p}=0.030)[40]$.

Increased CCA- IMT measurements have been employed to predict the extent and severity of CAD and have been found to be strongly associated with an increased risk of CV morbidity and mortality [41]. Similarly, reductions in CCAIMT thickness have been correlated with decreased CAD morbidity and mortality and used as an end point surrogate marker of atherosclerosis regression and dyslipidemias improvement in patients on lipid lowering therapy including SAIs [42]. There is need to identify sub-clinical CAD using atherosclerosis surrogate markers like CCA-IMT in SAIs that will help identify high-risk population for early preventive strategies to reduce future risk of CAD.

Normal CCA-IMT values have been defined on the basis of age and gender distribution curves within a general healthy population, as reported in the ARIC study [43]. The ASE consensus statement concludes that CCA-IMT values 
that are $\geq 75$ th percentile suggest risk higher than that predicted by the FRS and should be regarded as "high" values [24]. Values that fall within the 25 th to 75 th percentile range are considered "average" and should not affect traditional risk estimates. Values that are $\leq 25$ th percentile are "low" and suggest risk lower than that predicted by the FRS. One of the biggest challenge faced by cardiologists and vascular physicians is how best to interpret the results of trials using CCAIMT as the primary end point. Another challenge is how to integrate new imaging measures such as CCA-IMT into clinical practice. The ASE concluded that measuring CCAIMT and identifying carotid plaque may be useful in evaluating $\mathrm{CV}$ risk in the following patient populations [24]; (1) patients with intermediate cardiovascular disease risk (6\% to $20 \% 10$-year risk for myocardial infarction by the FRS), (2) patients with family histories of premature CVDs in firstdegree relatives; (3) patients aged $<60$ years with severe single-risk factor abnormalities (i.e., genetic dyslipidemia), who would otherwise not be candidates for pharmacotherapy; and (4) women aged $<60$ years with $\geq 2$ cardiovascular disease risk factors. In addition, consensus statement also suggests that CCA-IMT can be used if the "burden of subclinical vascular disease" is unclear or if evaluation for the degree of aggressiveness of therapy is needed [24] CCAIMT measurement has not been recommended for patients in whom the results would not change treatment, such as patients with established CAD.

It is important to recognize that current recommendations for the clinical use of CCA-IMT are based on observational studies. The use of CCA-IMT ultrasound measurement visualizes the long-term effects of different risk factors on the arterial wall and on the development of atherosclerotic changes in a given patient. It allows the presence of atherosclerosis to be detected almost directly and, to some extent, semi-quantitatively. Due to slow changes, CCA-IMT demonstrates the "overall atherosclerotic burden" and reflects the risk of death and of other $\mathrm{CV}$ events, even when classic risk factors are successfully corrected by therapeutic interventions. Ultimately, prospective trials comparing the effectiveness of CCA-IMT as a predictive tool of CV risk with that of other novel markers would best direct clinical recommendations for this imaging measure

\section{INFLAMMATORY BIOMARKERS}

SAIs have higher CAD morbidity and mortality rates and conventional risk factors do not explain this excess risk. Thus, much attention has focused on whether plasma levels of circulating markers of vascular inflammation may help identify SAIs at high risk for future CV events. Inflammation plays a central role in mediating all phases of atherosclerosis, from initial recruitment of circulating leucocytes to the arterial wall to the eventual rupture of the unstable plaque. I hereby present recent updates on C-reactive protein (CRP) and dysfunctional HDL with special focus on SAIs in determining vascular events and the potential use of inflammatory bio-markers for cardiovascular risk prediction.

\section{C - Reactive Protein (CRP)}

C-reactive protein (CRP) is a classical acute phase reactant and a member of the pentraxin family of innate immune response proteins $[44,45]$. CRP is mainly produced in the liver in response to interlukin 6 IL- 6 and thus has been thought of as an inactive downstream, by-stander marker of the inflammatory cascade. However, recent data suggest that CRP may play a direct role in atherogenesis. CRP is a prototypic marker of inflammation. Numerous prospective studies in healthy volunteers have confirmed that high-sensitivity CRP (hsCRP) predicts CV events, and hsCRP seems additive to an elevated total cholesterol level and a total/highdensity lipoprotein (HDL) cholesterol ratio in men and women [46]. In smokers and people with metabolic syndrome, hsCRP levels are elevated; in elderly people, there seems to be a relationship between hsCRP and CV events and mortality [47]. Several properties of CRP make it proatherogenic; however; pending further studies, it should be considered as a risk marker [48]. In people with acute coronary syndromes, hsCRP measurement may be valuable [46]. Elevated levels in the highest quantile seem to predict greater mortality and poorer prognosis in patients with unstable angina and MI. While hsCRP is a strong independent predictor of risk of future MI, stroke, peripheral arterial disease, and vascular death, the validity of hsCRP as a risk marker needs to be assessed in all populations. Weight loss, statin drugs, aspirin, and high-dose alpha tocopherol therapy could affect hsCRP [48]. It has its greatest validity as an adjunctive measure in the primary prevention of cardiovascular disease. Data from two large statin trials suggest that testing for CRP may identify many patients without hyperlipidaemia who are at high risk for future cardiovascular events and who may benefit from statin therapy [49]. Once confirmed in ongoing large-scale prospective trials, screening for inflammation using CRP as a biomarker could prove an important adjunctive method for identifying individuals at increased risk who would benefit most from targeted preventive interventions. Research on CRP is even more relevant in South Asians in general and SAIs in particular, since they are at high risk for the development of metabolic syndrome, type 2 diabetes and CAD [50]. Sub-clinical inflammation is a recent addition to the long list of risk factors in SAIs. CRP levels in SAIs were reported to be higher than in Europeans [51, 52]. However, the precise significance of high CRP levels in SAIs is not known and definitive answers would be provided by the prospective studies correlating CRP levels in SAIs to the CV endpoints and glucose intolerance in long-term prospective studies. Further, whether high CRP levels alone in young SAIs would influence future CV risk is not known. In summary, emerging evidence suggests that CRP is a robust marker for the prediction and prognosis of CAD and type 2 diabetes however its impact remains to be ascertained. However, the significance of elevated CRP levels as a marker for $\mathrm{CAD}$ in populations exposed to repeated and persistent infections in childhood is not clear. There is ongoing debate about the added value of measuring CRP concentrations to improve risk prediction, and that it may be premature.

\section{Dysfunctional HDL (Dys-HDL)}

High density lipoprotein (HDL) is a heterogeneous lipoprotein, containing several surface apolipoproteins (Apo AI, AII, AIV, C, E, J, and D). Apolipoprotein A-I (Apo A-I) is the principle protein of HDL, which also carries enzymes, such as paraoxonase 1 (PON 1), platelet activating factor (PAF) - acetyl hydrolase, lecithin cholesterol acyl transferase (LCAT), and cholesteryl ester transfer protein (CETP). Dif- 
ferences in HDL particle size result mainly from the number of apolipoprotein molecules and the volume of the cholesterol ester in the core of the particle [53].

1. HDL has antioxidant, anti-inflammatory, and antithrombotic properties that contribute to its function as an anti-atherogenic agent. Although our understanding of how HDL protects against CAD is still incomplete, evidence supports at least three major athero-protective mechanisms of HDL.

2. HDL is an integral component of the reverse cholesterol transport process, functioning as a carrier of excess cellular cholesterol from peripheral tissues to the liver, where it is excreted from the body as bile acids and cholesterol. More specifically, HDL mediates efflux of cholesterol from cholesterol-loaded macrophages by passive diffusion, through scavenger receptor B1 (SR B1), and, most significantly, via the protein Apo A-I [54, 55]. Apo A-I functions through an ATP-binding cassette transporter A1 (ABCA1) in the vessel wall, where it accepts free cholesterol, forming pre-beta HDL that matures after esterification to cholesteryl esters (CE) and then by lecithin-cholesterol acyltransferase to alpha migrating HDL [55].

3. A series of antioxidant enzymes which protect LDL from oxidation are associated with HDL. Oxidized lipids are transferred to HDL from LDL and are hydrolyzed by HDL-associated PON1, LCAT, and platelet activating factor (PAF) acetylhydrolase enzymes [56-58].file:///C:/ HDL study/understanding hDL.htm - R26-3425 The activities of these enzymes destroy oxidized lipids and also inhibit their formation. Removing the oxidized lipids initiates a positive feedback loop that results in further activation of the enzymes and further destruction of the oxidized lipids. In addition, Apo A-I reduces lipid peroxides within LDL, independent of PON1 [59].

HDL may protect against CAD by selectively decreasing endothelial cell adhesion molecules, which facilitate the binding of mononuclear cells to the vessel wall and promote lesion development [60].

However, according to several recent studies, in patients with CAD, HDL is not only ineffective as an antioxidant but, paradoxically, appears to be pro-oxidant, as assessed by its lipid peroxide content [61-64]. In addition, HDL from patients with a history of CAD enhances the oxidation of LDL and of phospholipids in LDL [62]. This pro-inflammatory HDL, which is dysfunctional, accumulates oxidants that inhibit HDL-associated antioxidant enzymes, render Apo A-I unable to promote ABCA1 mediated cholesterol efflux, and promotes the formation of LDL-derived oxidized lipids. What makes HDL become dysfunctional is not clearly understood, however it has been hypothesized that a certain variant of Apo A-I susceptible to oxidation and nitration could be the culprit [65]. Current data indicates that a $1 \%$ increase in HDL serum concentration can decrease cardiovascular risk by $2-3 \%$, independent of LDL levels [53]. However, HDL can have this protective effect only if it is functional. The incidence of dys-HDL in SAIs is not known, however several of the recent studies have shown prevalence of Dys-HDL in SAIs upto $50 \%$ in a group without CAD [40]. Moreover, small available data have shown Dys-HDL association with CCA-IMT [37]. Considering Apo-A-I polymorphisms as a hypothesized cause of Dys-HDL, data in SAIs found six novel single nucleotide polymorphisms (SNPs) in Apo-A-I gene. One of those showed association with low HDL in SAIs [65], however association of Apo-A-I polymorphism with Dys-HDL is still work under progress and needs larger prospective studies.

\section{CONCLUSIONS}

Given that SAIs are known to carry a disproportionately high risk for CAD and that traditional CAD risk factors may not fully explain the excess risk, there is a need to explore and understand other non-traditional risk factors. SAIs are under-represented in major clinical trials, and evidencebased management strategies of CAD in this population are lacking. Most clinicians are aware of the low HDL levels in this group, but whether this is due to isolated low HDL levels, high total cholesterol/HDL ratio, or an elevated nonHDL level, is not known. Moreover, the quality of HDL and its role in CAD protection is rapidly emerging. The time has now come for $\mathrm{CAD}$ to be considered the number one public health problem in SAIs, the second largest Asian immigrant population in the US. Emerging data from some wellplanned community-based investigations have emphasized the gravity of this rapidly increasing epidemic. The good news is that the epidemiologic studies have already shown that the bulk of the CAD can be prevented or at least its manifestations can be delayed. A multidisciplinary approach towards improving lifestyle methods involving the population at risk, healthcare personnel, and the government is required to diminish the incidence. From SAIs' perspective, there is a need for implementation of newer guidelines as well as lowering the threshold for initiating therapeutic interventions. IMT may be helpful in predicting the occurrence of CV events in SAs that are asymptomatic but at a high risk due to additional factors after controlling for factors such as sex, age, any other clinical conditions and drug treatment the patient may be undergoing. IMT would be a good tool for therapeutic intervention, for monitoring the efficacy of statins, ACE inhibitors and other drugs in SAs. However, the assessment needs to take into account long term effects in IMT for the same patient, to control for any pre-existing conditions that may cause inflammation [66]. With respect to the potential value of novel risk factors for $\mathrm{CV}$ risk prediction, we illustrate why this work is very much in its infancy and currently not guaranteed to reach clinical utility. Indeed, the existence of several more powerful and easily measured predictors of diabetes, suggests that the additional value of novel markers may be limited. Ultimately, prospective trials comparing the effectiveness of CCA-IMT as a predictive tool of CV risk with that of other novel markers would best direct clinical recommendations for this imaging measure. Nevertheless, several suggestions to improve relevant research are given. CCA-IMT is a noninvasive surrogate marker of atherosclerosis and proven to be helpful in detecting sub-clinical CAD by stratifying populations at highest risk for CAD. In addition, determining the presence of DysHDL in SAIs will answer several questions related to the presence of altered HDL level and function. This information will not only help to stratify this high risk asymptomatic group, but will also be useful from a disease management point of view. 


\section{REFERENCES}

[1] Uppaluri CR. Heart disease and its related risk factors in Asian Indians. Ethn Dis 2002; 12: 45-53.

[2] Joshi P, Islam S, Pais P, et al. Risk factors for early myocardial infarction in South Asians compared with individuals in other countries. JAMA 2007; 297: 286-94.

[3] Enas EA, Garg A, Davidson MA, Nair VM, Huet BA, Yusuf S. Coronary heart disease and its risk factors in first-generation immigrant Asian Indians to the United States of America. Indian Heart J 1996; 48: 343-53.

[4] Enas EA, Mehta J. Malignant coronary artery disease in young Asian Indians: thoughts on pathogenesis, prevention, and treatment. Clin Cardiol 1995; 18: 131-5.

[5] Enas EA, Yusuf S, Mehta J. Prevalence of coronary artery disease in Asian Indians. Am J Cardiol 1992; 70: 945-9.

[6] Mohan V, Deepa R, Rani SS, Premalatha G. Chennai Urban Population Study (CUPS No.5). Prevalence of coronary artery disease and its relationship to lipids in a selected population in South India. The Chennai Urban Population Study (CUPS No. 5). J Am Coll Cardiol 2001; 38: 688-9.

[7] McKeigue PM, Marmot MG. Mortality from coronary heart disease in Asian communities in London. BMJ 1988; 297: 903.

[8] Cappuccio F, Cook D, Atkinson R, Strazzullo P. Prevalence, detection, and management of cardiovascular risk factors in different ethnic groups in south London. Heart 1997; 78: 555-63.

[9] Bhopal R, Hayes L, White M, Unwin N, Harland J, Ayis S. Ethnic and socio-economic inequalities in coronary heart disease, diabetes and risk factors in Europeans and South Asians. J Public Health Med 2002; 24: 95-105.

[10] Bhatnagar D, Anand IS, Durrington PN, et al. Coronary risk factors in people from the Indian subcontinent living in West London and their siblings in India. Lancet 1995; 345: 405-9.

[11] Patel JV, Vyas A, Cruickshank JK, et al. Impact of migration on coronary heart disease risk factors: comparison of Gujaratis in Britain and their contemporaries in villages of origin in India. Atherosclerosis 2006; 185:297-306.

[12] Patel S, Unwin N, Bhopal R, et al. A comparison of proxy measures of abdominal obesity in Chinese, European and South Asian adults. Diabet Med 1999; 16: 853-60.

[13] Din JN, Ashman OA, Aftab SM, Jubb AW, Newby DE, Flapan AD. Increased arterial stiffness in healthy young South Asian men. J Hum Hypertens 2006; 20: 163-5.

[14] Toutouzas P, Richter D. Carotid intima-media thickness (cIMT): a useful clinic tool or research luxury? Another view of the ENHANCE trial. Angiology 2008; 59 (2 Suppl): 77S-9S.

[15] Goyal A, Yusuf S. The burden of cardiovascular disease in the Indian subcontinent. Indian J Med Res 2006; 124: 235-44.

[16] Kuller LH, Velentgas P, Barzilay J, Beauchamp NJ, O'Leary DH, Savage PJ. Diabetes mellitus: sub-clinical cardiovascular disease and risk of incident cardiovascular disease and all-cause mortality. Arterioscler Thromb Vasc Biol 2000; 20: 823-9.

[17] Pasternak RC, Abrams J, Greenland P, Smaha LA, Wilson PW, Houston-Miller N. 34th Bethesda Conference: task force \#1-Identification of coronary heart disease risk: is there a detection gap? J Am Coll Cardiol 2003; 41: 1863-74.

[18] Devine PJ, Carlson DW, Taylor AJ. Clinical value of carotid intima-media thickness testing. J Nucl Cardiol 2006; 13: 710-8.

[19] Patel SN, Rajaram V, Pandya S, Fiedler BM, Bai CJ, Neems R. Emerging, noninvasive surrogate markers of atherosclerosis. Curr Atheroscler Rep 2004; 6: 60-8.

[20] Smith SC, Greenland P, Grundy SM. Prevention Conference V. Beyond Secondary Prevention: identifying the high-risk patient for primary prevention. Executive Summary. Circulation 2000; 101: 111-6.

[21] Egger M, Krasinski A, Rutt BK, Fenster A, Parraga G. Comparison of B-mode ultrasound, 3-dimensional ultrasound, and magnetic resonance imaging measurements of carotid atherosclerosis. J Ultrasound Med 2008; 27: 1321-34.

[22] Pignoli P, Tremoli E, Poli A, Oreste P, Paoletti R. Intimal plus medial thickness of the arterial wall: a direct measurement with ultrasound imaging. Circulation 1986; 77: 1399-406.

[23] O'Leary DH, Polak JF, Wolfson SK Jr, et al. Use of sonography to evaluate carotid atherosclerosis in the elderly. The Cardiovascular Health Study. CHS Collaborative Research Group. Stroke 1991; 22: 1155-63.
Stein JH, Korcarz CE, Hurst RT, et al. Use of carotid ultrasound to identify subclinical vascular disease and evaluate cardiovascular disease risk: a consensus statement from the American Society of Echocardiography Carotid Intima-Media Thickness Task Force. J Am Soc Echocardiography 2008; 21: 93-111.

[25] Howard G, Manolio TA, Burke GL, Wolfson SK, O'Leary DH. Does the association of risk factors and atherosclerosis change with age? An analysis of the combined ARIC and CHS cohorts. The Atherosclerosis Risk in Communities (ARIC) and Cardiovascular Health Study (CHS) investigators. Stroke 1997; 28: 1693-701.

[26] Chambless LE, Heiss G, Folsom AR, et al. Association of coronary heart disease incidence with carotid arterial wall thickness and major risk factors: the Atherosclerosis Risk in Communities (ARIC) Study, 1987- 1993. Am J Epidemiol 1997; 146: 483-94.

[27] Ebrahim S, Papacosta O, Whincup P, et al. Carotid plaque, intimamedia thickness, cardiovascular risk factors, and prevalent cardiovascular disease in men and women: the British Regional Heart Study. Stroke 1999; 30: 841-50.

[28] Bots ML, Breslau PJ, Briet E, et al. Cardiovascular determinants of carotid artery disease: the Rotterdam Elderly Study. Hypertension 1992; 19: 717-20.

[29] Hughes K, Yeo PP, Lun KC, et al. Cardiovascular diseases in Chinese, Malays, and Indians in Singapore. II. Differences in risk factor levels. J Epidemiol Commun Health 1990; 44: 29-35.

[30] National Cholesterol Education Program (NCEP) expert panel on detection, evaluation and treatment of high blood pressure in adults (ATP III). Third report of the National Cholesterol Education Program (NCEP) expert panel on detection, evaluation and treatment of high cholesterol in adults (ATP III) final report. Circulation 2002; 106: 3121-43.

[31] De Backer G, Ambrosioni E, Borch-Johnsen K, et al. European guidelines on cardiovascular disease prevention in clinical practice. Third Joint Task Force of European and Other Societies on Cardiovascular Disease Prevention in Clinical Practice. Eur Heart J 2003; 24: 1601-10.

[32] Yusuf S, Hawken S, Ounpuu S, et al. Effect of potentially modifiable risk factors associated with myocardial infarction in 52 countries (the INTERHEART study): case control study. Lancet 2004; 364: $937-52$.

[33] Ridker PM, Hennekens CH, Buring JE, Cook NR, Rifai N. Creactive protein and other markers of inflammation in the prediction of cardiovascular disease in women. N Engl J Med 2000; 342: 836-43.

[34] Teo KK, Ounpuu S, Hawken S, et al. Tobacco use and risk of myocardial infarction in 52 countries in the INTERHEART study: a case-control study. Lancet 2006; 368: 647-58.

[35] Coutinho M, Gerstein HC,Wang Y, Yusuf S. The relationship between glucose and incident cardiovascular events. A metaregression analysis of published data from 20 studies of 95,783 individuals followed for 12.4 years. Diabetes Care 1999; 22: 233-40.

[36] Jadhav UM, Kadam NN. Carotid intima-media thickness as an independent predictor of coronary artery disease. Indian Heart J 2001; 53: 458-62.

[37] Howard G, Sharrett AR, Heiss G, et al. Carotid artery intimalmedial thickness distribution in general populations as evaluated by B-mode ultrasound. ARIC Investigators. Stroke 1993; 24: 1297304.

[38] Dodani S, Marion L, Sharma G, Reed GL, George V. The association of carotid intima media thickness with dysfunctional HDL in South Asians. Arch Med Sci 2008; 4: 40-6.

[39] Dodani S, Kaur R, Reddy S, Reed GL, Navab M, George V. Can dysfunctional HDL explain high coronary artery disease risk in South Asians? Int J Cardiol 2008; 129: 125-32.

[40] Tang R, Hennig M, Bond MG, Hollweck R, Mancia G, Zanchetti A. Quality control of B-mode ultrasonic measurement of carotid artery intima-media thickness: the European Lacidipine Study on Atherosclerosis. J Hypertens 2005; 23: 1047-54.

[41] Touboul PJ, Hennerici MG, Meairs S, et al. Mannheim carotid intima-media thickness consensus (2004-2006). An update on behalf of the advisory board of the 3rd and 4th watching the risk symposium 13th and 15th European stroke conferences, Mannheim, Germany, 2004, and Brussels, Belgium, 2006. Cerebrovasc Dis 2007; 23: 75-8.

[42] Lorenz MW, Markus SH, Bots ML, Rosval M and Sitzer M. Prediction of clinical cardiovascular events with carotid intima-media 
thickness: a systematic review and meta-analysis. Circulation 2007; 115: 459-67.

[43] Chambless LE, Heiss G, Folsom AR, et al. Association of coronary heart disease incidence with carotid arterial wall thickness and major risk factors: the Atherosclerosis Risk in Communities (ARIC) study. Am J Epidemiol 1997; 146: 483-94.

[44] Du Clos TW. Function of C-reactive protein. Ann Med 2000; 32: 274-8.

[45] Lloyd-Jones DM, Liu K, Tian L, Greenland P. Narrative review: Assessment of C-reactive protein in risk prediction for cardiovascular disease. Ann Intern Med 2006; 145: 35-42.

[46] Cesari M, Penninx BW, Newman AB, et al. Inflammatory markers and onset of cardiovascular events: results from the Health $\mathrm{ABC}$ study. Circulation 2003; 108: 2317-22.

[47] Timpson NJ, Lawlor DA, Harbord RM, et al. C-reactive protein and its role in metabolic syndrome: mendelian randomisation study. Lancet 2005; 366: 1954-9.

[48] Blake GJ, Rifai N, Buring JE, Ridker PM. Blood Pressure, CReactive Protein, and Risk of Future Cardiovascular Events. Circulation 2003; 108: 2993-9.

[49] Sattar N, Murray HM, McConnachie A, et al. C-reactive protein and prediction of coronary heart disease and global vascular events in the Prospective Study of Pravastatin in the Elderly at Risk (PROSPER). Circulation 2007; 115: 981-9.

[50] Yusuf S, Reddy S, Ounpuu S, Anand S. Global burden of cardiovascular diseases: part II: variations in cardiovascular disease by specific ethnic groups and geographic regions and prevention strategies. Circulation 2001; 104: 2855

[51] Chambers JC, Eda S, Bassett P, et al. C-reactive protein, insulin resistance, central obesity, and coronary heart disease risk in Indian Asians from the United Kingdom compared with European whites. Circulation 2001; 104: 145

[52] Forouhi NG, Sattar N, McKeigue PM. Relation of C-reactive protein to body fat distribution and features of the metabolic syndrome in Europeans and South Asians. Int J Obes Relat Metab Disord 2001; 25: 1327

[53] Gordon DJ, Probstfield JL, Garrison RJ, et al. High-density lipoprotein cholesterol and cardiovascular disease. Four prospective American studies. Circulation 1989; 79: 8-15.

[54] Yancey PG, Bortnick AE, Kellner-Weibel G, de la Llera-Moya M, Phillips MC, Rothblat GH. Importance of different pathways of cellular cholesterol efflux. Arterioscler Thomb Vasc Biol 2003; 23 : $712-9$
[55] Williams DL, Connelly MA, Temel RE, et al. Scavenger receptor B1 and cholesterol trafficking. Curr Opin Lipidol 1999; 10: 32939 .

[56] Takahashi Y, Smith JD. Cholesterol efflux to apolipoprotein AI involves endocytosis and resecretion in a calcium-dependent pathway. Proc Natl Acad Sci USA 1999; 96: 11358-63.

[57] Remaley AT, Stonik JA, Demosky SJ, et al. Apolipoprotein specificity for lipid efflux by the human ABCA1 transporter. Biochem Biophys Res Commun 2001; 280: 818-23.

[58] Mackness MI, Arrol S, Abbott C, Durrington PN. Protection of low-density lipoprotein against oxidative modification by highdensity lipoprotein associated paraoxonase. Atherosclerosis 1993 104:129-35.

[59] Marathe GK, Zimmerman GA, McIntyre TM. Platelet-activating factor acetylhydrolase, and not paraoxonase-1, is the oxidized phospholipid hydrolase of high density lipoprotein particles. J Biol Chem 2002; 278: 3937-47.

[60] Barter PJ, Baker PW, Rye KA. Effect of high-density lipoproteins on the expression of adhesion molecules in endothelial cells. Curr Opin Lipidol 2002; 13: 285-8.

[61] Navab M, Anantharamaiah GM, Reddy ST, Van Lenten BJ, Ansell BJ, Fogelman AM. Mechanisms of disease: proatherogenic HDL-an evolving field. Nat Clin Pract Endocrinol Metab 2006; 2: 504 11 .

[62] Ansell BJ, Fonarow GC, Fogelman AM. High-density lipoprotein: is it always atheroprotective? Curr Atheroscler Rep 2006; 8: 40511 .

[63] Ansell BJ, Fonarow GC, Fogelman AM. High-density lipoprotein function recent advances. J Am Coll Cardiol 2005; 46: 1792-8.

[64] Navab M, Ananthramaiah GM, Reddy ST, et al. The double jeopardy of HDL. Ann Med 2005; 37: 173-8.

[65] Zheng L, Nukuna B, Brennan ML, et al. Apolipoprotein A-I is a selective target for myeloperoxidase-catalyzed oxidation and functional impairment in subjects with cardiovascular disease. J Clin Invest 2004; 114: 529-41.

[66] Dodani S, Dong Y, Zhu H, George V. Can novel Apo A-I polymorphisms be responsible for low HDL in South Asian immigrants Indian J Hum Genet 2008; 14: 9-15.

[67] Gasparyan AY. The Use of Carotid Artery Ultrasonography in Different Clinical Conditions. Open Cardiovasc Med J 2009; 3: 7880 